\title{
ELECTROSPINNING AURICULAR SHAPED SCAFFOLDS FOR TISSUE ENGINEERING
}

\author{
Jochen Walser $^{1}$, Marco D. Caversaccio ${ }^{2}$, Stephen J. Ferguson ${ }^{1}$ \\ ${ }^{1}$ Institute for Biomechanics, ETH Zurich, Switzerland \\ ${ }^{2}$ Inselspital, University of Bern, Switzerland \\ jowalser@ethz.ch
}

\begin{abstract}
Poly( $(\varepsilon)$ caprolactone scaffolds have been electrospun directly into an auricular shaped conductive mould. Bovine chondrocytes were harvested from articular cartilage and seeded onto 16 of the produced scaffolds, which received either an ethanol (group A) or a plasma treatment (group B) for sterilisation before seeding. The seeded scaffolds were cultured for 3 weeks in vitro and analysed with regard to total DNA and GAG content as well as the expression of $A G G, C O L 1, C O L 2, M M P 3$ and $M M P 13$. Rapid cell proliferation and GAG accumulation was observed until week 2. However, total DNA and GAG content decreased again in week 3. qPCR data shows a slight increase in the expression of anabolic genes and a slight decrease for the catabolic genes, with a significant difference between the groups $A$ and $B$ only for COL2 and MMP13.
\end{abstract}

Keywords: Electrospinning, Tissue Engineering, Auricle

\section{Introduction}

The reconstruction of auricular cartilage remains a significant challenge, as there is still no established method which allows forming patient patient-specific tissue engineered neo-cartilage [1], [2]. Electrospinning is a method to produce polymer fibre mats with fibre diameters ranging from $200 \mathrm{~nm}$ up to $10 \mu \mathrm{m}$ and has been shown to have a potential for tissue engineering applications [3]. A polymer solution is charged using a potential of 20-30 kilovolts at the needle tip of a syringe. In this range, electrostatic repulsion exceeds surface tension of the polymer solution in the droplet and a small polymer jet follows the electric field lines and is drawn to a collector, which is connected to ground potential. Xue et al [4] recently reported the production of neo-cartilage from flat e-spun sheets, which were pressed into an auricular shaped form and cultured for several weeks in vitro and in vivo. This study investigates the possibility of directly spinning into a conductive mould and its potential for cartilage tissue engineering.

\section{Methods}

Auricular shaped mould: An auricular shaped mould has been designed by adapting an artificial 3D ear model with SolidWorks 2011 (Dassault Systems, France). The size of the mould was reduced to $50 \%$ of that of a human ear, rapid-manufactured from stainless steel by selective laser melting and mounted as the collector electrode onto the electrospinning device.
Scaffold production and treatment: 16 auricular shaped scaffolds were electrospun into the described moulds from $2.5 \mathrm{ml}$ of a $8 \mathrm{w} \%$ poly $(\varepsilon)$ caprolactone $\left(\mathrm{PCL}, \mathrm{M}_{\mathrm{w}}=80000\right)$ solution dissolved in a 1:6 mixture of methanol and chloroform. The scaffolds were spun with an electrode potential of $25 \mathrm{kV}$, at a gap distance of $20 \mathrm{~cm}$ and a feed rate of $0.03 \mathrm{ml} / \mathrm{min}$. The collector mould was manually repositioned multiple times during the spinning process in order to fill all parts of the mould. After the spinning and demoulding, all spun scaffolds were degassed for several days in a desiccator. The 16 ears were split randomly into 2 groups. Group A was disinfected for several days in $80 \%$ Ethanol, while group B was plasma sterilised. All scaffolds were kept in DMEM until the start of the cell culture.

Cell culture: Bovine chondrocytes were harvested from articular cartilage of a 6 month old calf, digested with $0.4 \%$ Pronase for $90 \mathrm{~min}$ and $0.025 \%$ Collagenase P for 17 hours. $2 \times 10^{6}$ of the released chondrocytes were then seeded on each ear at a concentration of $2 \times 10^{6}$ cells $/ \mathrm{ml}$ and cultured for 3 weeks. The culture medium consisted of DMEM, 10\% FCS, Vit. C. for the first week and DMEM, 5\% FCS, Vit. C. for the following 2 weeks. 2 ears per group were taken each time at day 1, 7, 14 and 21 for DNA, Glycosaminoglycan (GAG) and qPCR analysis. An exchange of culture medium was performed every 3-4 days.

GAG/DNA and qPCR measurements: Each ear was cut into 6 pieces, where 3 of these were used for the quantification of total GAG and DNA content and normalized to specimen weight. The second half was used for gene expression analysis of Aggrecan (AGG), Colagen type I and type II (COL1/2), matrix metalloproteinase 3 and 13 (MMP3/13) in reference to GAPDH as housekeeping gene and day 1.

Statistical analysis: An analysis of variance (ANOVA) including a post-hoc Tukey test was conducted for GAG/DNA measurements as well as for gene expression data in order to test for significant differences between the groups and the sampling days respectively.

\section{Results}

The PCL fibres could be successfully deposited within the mould and the resulting scaffolds had a thickness of about $1 \mathrm{~mm}$. However, bridging was observed at small gaps of the mould. SEM imaging revealed a different morphology of the fibre layer first deposited in the mould (bottom layer) compared to the top layer (Figure 1). Rapid cell proliferation and ECM production was observed in both 
groups, while the plasma group had a 3 times higher cell count and 6 times more ECM for the first 2 weeks compared to the ethanol group. At Day 21 a heavy loss of DNA for was observed for the plasma group as well as a loss of GAG content for both groups (Table 1).

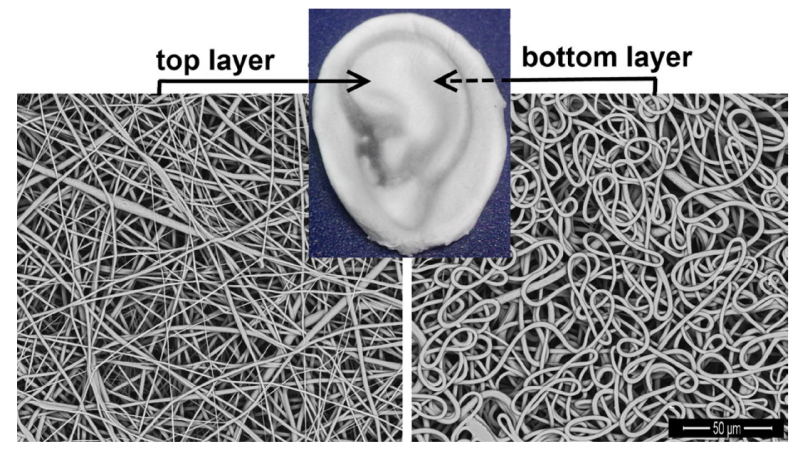

Figure 1: Electrospun ear scaffold and its morphology

Table 1: Total DNA and GAG content of produced constructs, normalized to specimen weight.

\begin{tabular}{ccccc}
\hline & \multicolumn{2}{c}{ DNA $[\mathbf{n g} / \mathbf{m g}]$} & \multicolumn{2}{c}{ GAG $[\boldsymbol{\mu g} / \mathbf{m g}]$} \\
Day & Ethanol & Plasma & Ethanol & Plasma \\
\hline 1 & $3.3 \pm 0.9$ & $12.1 \pm 4.3$ & - & - \\
7 & $16.7 \pm 5.1$ & $40.3 \pm 18.3$ & $0.39 \pm 0.14$ & $2.45 \pm 0.20$ \\
14 & $20.2 \pm 6.8$ & $69.9 \pm 24.7$ & $1.14 \pm 0.69$ & $6.32 \pm 4.24$ \\
21 & $23.3 \pm 7.3$ & $33.8 \pm 8.7$ & $0.54 \pm 0.38$ & $3.13 \pm 1.09$ \\
\hline
\end{tabular}

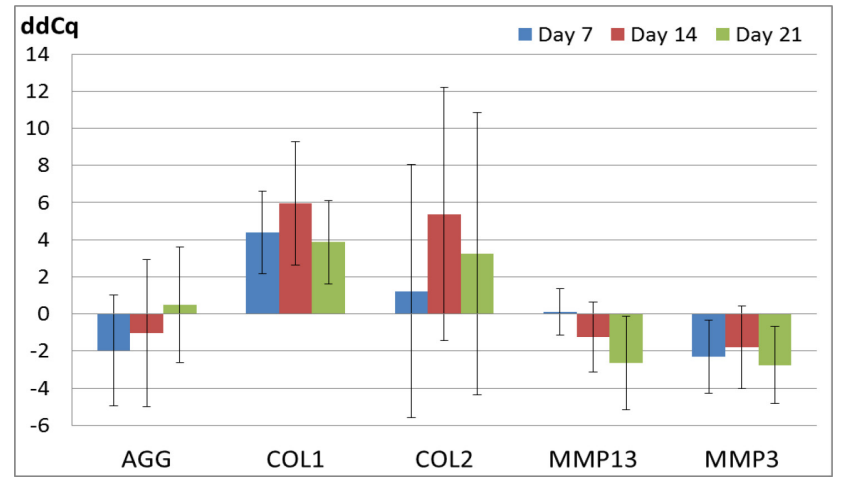

Figure 2: Expression of AGG, COL1, COL2, MMP3 and MMP13 in reference to GAPDH and day1 for the ethanol treated ear scaffolds

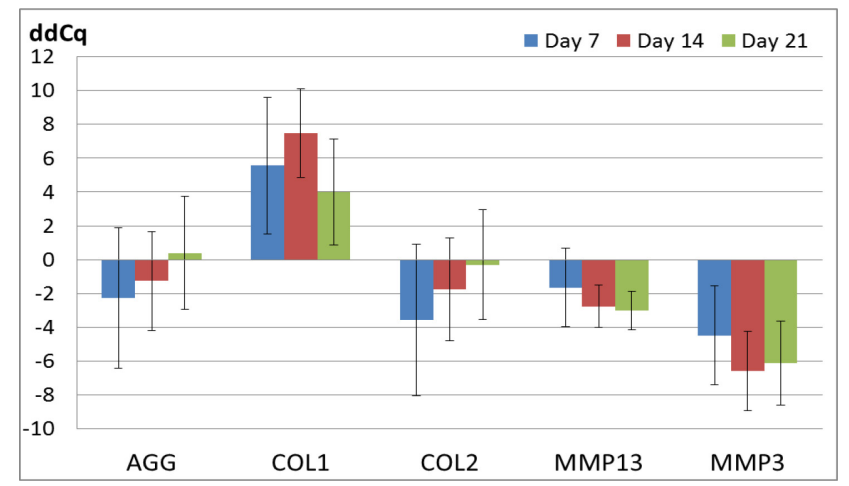

Figure 3: Expression of AGG, COL1, COL2, MMP3 and MMP13 in reference to GAPDH and dayl for the plasma treated ear scaffolds
Quantitative real time PCR data draws a mixed picture. Concerning the anabolic genes, AGG and Col2 show no clear trend and high standard deviation while Coll basically reflects the results of the total GAG content (Figure 2 and Figure 3). On the catabolic side, MMP3 and MMP13 show a trend to be down-regulated for both groups. However statistical analysis reveals only a significant difference in Col2 $(\mathrm{p}<0.05)$ and MMP13 $(\mathrm{p}<0.001)$ between the ethanol and the plasma treated scaffolds.

\section{Discussion}

As reflected by the standard deviation, there was a huge variation even between different sections of the same ears. Evidence suggests that the chondrocytes were not evenly distributed on the scaffold and hence would respond differently regarding cell proliferation, ECM production and gene expression depending on cell location and density. Further investigation will be necessary and the cell seeding procedure has to be critically evaluated. An increase in sample number might also be necessary for a follow-up study in order to minimise the influence of these variations. Furthermore the plasma sterilization in particular might have caused not only a surface activation of the fibres but also changes in scaffold morphology. Nevertheless, rapid cell proliferation and ECM production underline the potential of these constructs for cartilage tissue engineering.

\section{Acknowledgement}

Technical assistance of S. Dudli is gratefully acknowledged. This study is part of the NCCR Co-Me project "Cartilage Tissue Engineering for ORL Applications" and funded by the Swiss National Science Foundation.

\section{Bibliography}

[1] D. A. Bichara, N.-A. O'Sullivan, I. Pomerantseva, X. Zhao, C. A. Sundback, J. P. Vacanti, and M. A. Randolph, "The tissue-engineered auricle: past, present, and future.," Tissue engineering. Part B, Reviews, vol. 18, no. 1, pp. 51-61, Feb. 2012.

[2] A. Sterodimas, J. de Faria, W. E. Correa, and I. Pitanguy, "Tissue engineering and auricular reconstruction: a review.," Journal of plastic, reconstructive \& aesthetic surgery: JPRAS, vol. 62, no. 4, pp. 447-52, Apr. 2009.

[3] D. R. Nisbet, J. S. Forsythe, W. Shen, D. I. Finkelstein, and M. K. Horne, "Review paper: a review of the cellular response on electrospun nanofibers for tissue engineering.," Journal of biomaterials applications, vol. 24, no. 1, pp. 7-29, Jul. 2009.

J. Xue, B. Feng, R. Zheng, Y. Lu, G. Zhou, W. Liu, Y. Cao, Y. Zhang, and W. J. Zhang, "Engineering ear-shaped cartilage using electrospun fibrous membranes of gelatin/polycaprolactone.," Biomaterials, vol. 34, no. 11, pp. 2624-31, Apr. 2013. 